\title{
Tackling nonadherence in psychiatric disorders: current opinion
}

This article was published in the following Dove Press journal:

Neuropsychiatric Disease and Treatment

12 June 2014

Number of times this article has been viewed

\author{
Saeed Farooq ${ }^{1,2}$ \\ Farooq $\mathrm{Naeem}^{3}$ \\ 'Staffordshire University, Staffordshire, \\ UK; ${ }^{2}$ Postgraduate Medical Institute, \\ Lady Reading Hospital, Peshawar, \\ Pakistan; ${ }^{3}$ Department of Psychiatry, \\ Queen's University, Kingston, Ontario, \\ Canada
}

Correspondence: Saeed Farooq Postgraduate Medical Institute, Lady Reading Hospital, Peshawar, Pakistan Email sfarooqIrh@yahoo.com

\begin{abstract}
Nonadherence to treatment is a major challenge in all fields of medicine, and it has been claimed that increasing the effectiveness of adherence interventions may have far greater impact on the health of the population than any improvement in specific medical treatments. However, despite widespread use of terms such as adherence and compliance, there is little agreement on definitions or measurements. Nonadherence can be intermittent or continuous, voluntary or involuntary, and may be specific to single or multiple interventions, which makes reliable measurement problematic. Both direct and indirect methods of assessment have their limitations. The current literature focuses mainly on psychotic disorders. A large number of trials of various psychological, social, and pharmacologic interventions has been reported. The results are mixed, but interventions specifically designed to improve adherence with a more intensive and focused approach and interventions combining elements from different approaches such as cognitive-behavioral therapy, family-based, and community-based approaches have shown better outcomes. Pharmacologic interventions include careful drug selection, switching when a treatment is not working, dose adjustment, simplifying the treatment regimen, and the use of long-acting injections. The results for the most studied pharmacologic intervention, ie, long-acting injections, are far from clear, and there are discrepancies between randomized controlled trials, nationwide cohort studies, and mirror-image studies. Nonadherence with treatment is often paid far less attention in routine clinical practice and psychiatric training. Strategies to measure and improve adherence in clinical practice are based more on personal experience than on research evidence. This overview focuses on strategies used for improving treatment adherence in psychiatric disorders in the light of current evidence, with emphasis on public health aspects of treatment adherence and the management of nonadherence in routine clinical practice.
\end{abstract}

Keywords: depression, anxiety, psychosis, compliance, drug treatment, clinical practice

\section{Introduction}

Nonadherence with treatment is a major public health challenge. In medicine, where practitioners are not faced with challenges such as lack of insight, almost half of interventions to improve treatment adherence are likely to fail. ${ }^{1}$ Therefore, a substantial number of patients do not get the required benefit of medical treatment, which results in poor health outcomes and increased health care costs. In the USA, it is estimated that the cost of nonadherence could be up to 300 billion dollars per year. ${ }^{2}$ It has been claimed that increasing the effectiveness of adherence interventions may have a far greater impact on the health of the population than any improvement in specific medical treatments. ${ }^{1}$ 
This overview focuses on strategies used for improving treatment adherence in psychiatric disorders in the light of current evidence. We also focus on public health aspects of treatment adherence in psychiatry. We do not review the evidence for effectiveness because this has been amply documented, and instead review the nature of these interventions and how they can be used in routine clinical practice.

Nonadherence figures vary widely, which is understandable in view of the different populations, variety of diagnoses, variable follow-up periods, and, most importantly, the different definitions and measurement methods used in research. In a systematic review, Lacro et a ${ }^{3}$ found that the nonweighted mean frequency of nonadherence was $40.5 \%$ (median $40 \%$, range $4 \%-72 \%$ ). Rates of nonadherence vary between $24 \%$ and $40 \%$ based on medication refill rates available from pharmacy records. ${ }^{4,5}$ Generally, studies that do not rely only on patient recall and use robust methods, such as pill count, electronic monitoring, and blood drug levels, tend to indicate higher rates of nonadherence. ${ }^{6,7}$ The degree of adherence also tends to vary widely over time. Valenstein et $\mathrm{al}^{8}$ observed that $61 \%$ of patients had adherence problems at some point over a 4 -year period of follow-up. However, only about $18 \%$ had consistently poor adherence during the same time period. It is estimated that $20 \%-60 \%$ of people suffering from bipolar disorder are nonadherent with prescribed medications at any given time, with a mean rate of $40 \% .{ }^{9}$ A longitudinal study found that, among patients who started lithium treatment, the median time to discontinuation was only 76 days. ${ }^{10}$ Alarmingly, it has been reported that nearly half of medical outpatients who are receiving antidepressant medication discontinue treatment during the first month. ${ }^{11}$

Unlike naturalistic studies, controlled trial settings allow us to assess adherence in a prospective manner, often with more accurate methods such as pill counts or blood levels. In addition, the characteristics of patients (including sociodemographic, diagnostic, and biological variables) are known in detail, making it easier to examine potential predictors of nonadherence. In randomized controlled trials, the risk of selection bias is always a problem as far as adherence is concerned, because patients recruited into trials are required to undergo consenting procedures. Therefore, they are more likely to adhere to the trial procedures and medication. Moreover, participation in a controlled trial alters the delivery and experience of treatment. Patients in clinical trials are also more likely to receive more attention than those in routine care. This includes assessments for trial outcomes, measures of adherence, reminders to attend clinical/research assessment sessions, and usually provision of medication as part of the trial. Further, adherence is often measured only among patients who continue in the trial, whereas patients who are nonadherent are counted as study dropouts. Patients who drop out from studies because of nonadherence are often reported as "withdrew consent" or "patient decision", and the underlying reasons are rarely examined in detail. Therefore, it is fair to assume that adherence is much greater in clinical trials than in routine care.

However, nonadherence with treatment is often paid far less attention in routine clinical practice and in the psychiatric literature. Terms such as adherence and compliance with medication remain in widespread use, but there is little agreement on definitions, measurements, and more importantly, how we assess these in routine clinical practice. There are no reliable tools which can assess medication adherence for common psychiatric disorders in the clinic. The current literature focuses mainly on psychotic disorders. This is surprising considering the prevalence of depression and its impact on health, well-being, and health care costs. It has been suggested that nearly half of medical outpatients who receive an antidepressant prescription discontinue treatment during the first month. ${ }^{11}$ In resource-poor settings, where the cost of medication is a major barrier to treatment adherence, even providing free or subsidized treatment does not always ensure treatment continuation. ${ }^{12}$ Discontinuation rates within 3 months of the start of treatment can reach $68 \%$, depending on the population studied and the agent used. ${ }^{13}$ Other studies have found that less than $33 \%$ of patients diagnosed with depression receive and take their medications appropriately, and only $40 \%$ reach an adequate dosage and duration of treatment. ${ }^{14}$ However, there is a dearth of studies addressing nonadherence in nonpsychotic disorders.

\section{Measurement and concept of adherence}

It is important to realize that nonadherence is a complex phenomenon and not a unitary concept. There is no agreement on an optimal level of adherence with treatment, given that $100 \%$ adherence with treatment is ideally required but rarely achieved in practice. Nonadherence can be intermittent or continuous. It can also be voluntary, ie, deliberately not taking medication due to lack of insight, or can be involuntary when the patient forgets or does not follow instructions due to poor understanding. Nonadherence may be specific to a single drug or to multiple medications advised by the physician.

Taking medication and reporting this to the physician is a voluntary act and can rarely be objectively verified. Therefore, 
definitions and measurement pose a major challenge. The recall measures commonly used in practice and research are likely to be inaccurate. The most potentially informative strategies depend upon monitoring treatment electronically, but these techniques are mostly invasive and/or expensive. ${ }^{1}$

It is difficult to classify methods for measuring nonadherence, but two broad groups, ie, direct and indirect, can be identified. Direct methods involve observing patients while they swallow their medication. This has been one of the most studied methods in medicine. An example is direct observation of treatment, as used in the DOTS (Directly Observed Treatment, Short course) program, which is often used in tuberculosis control and was recently adopted for schizophrenia. ${ }^{12}$ Other methods include measurement of drug concentrations in blood or other body fluids or measuring a biologic marker added to the medicine for this purpose.

Indirect methods of monitoring adherence include a number of techniques and assessment procedures. Simple methods include asking the patient, pill counts, and electronic devices that monitor the opening of a lid or tablet strip. ${ }^{15}$ The patient's account of medication-taking is easiest but is often unreliable. A simple measure is asking the patient about medication intake over a specified period. Adherence can be assessed at interview using a simple questionnaire, such as that used by Herz et al, ${ }^{16}$ which measures medicine adherence on a 5-point scale (1, always; 5, never). More recently, a novel technology, referred to as a digital health feedback system, ${ }^{15}$ has been employed. In this study, 28 adults with bipolar disorder and schizophrenia utilized a digital health feedback system (DHFS) which is a digital tablet, consisting of an ingestion sensor embedded in the tablet. The medicine is coated with an "ingestible event marker", which upon contact with gastric fluid electrolytes emits a unique signal. This signal is transmitted through bodily tissue to a small receiver worn in a patch on the torso which then transmits a signal to a cell phone indicating the time (and date) that the medication has been ingested. A pilot study showed that this approach was feasible and acceptable to patients, as 27 of 28 subjects $(96 \%)$ completed the study and the mean adherence rate was $74 \%{ }^{15}$

All these methods have their limitations. Even apparently accurate methods, such as observing patients while they are taking their medicine may have pitfalls, in that patients may appear to take the medicine but do not swallow it. Technologically advanced methods increase the reliability of assessment but are often too expensive to be used in routine clinical practice, and may erode the confidence of patients to play a more active role in management of their disorder.

\section{Tackling nonadherence in psychiatry}

The methods used to improve treatment adherence in psychiatry can be divided in two broad groups, ie, psychosocial and pharmacologic interventions, which are described briefly here.

\section{Psychosocial interventions}

These interventions refer to a broad range of psychologic and social measures which aim to modify patient behavior and encourage compliance with treatment, and there is significant overlap across different interventions. Psychosocial interventions are underpinned by different theoretical backgrounds, such as cognitive-behavioral therapy (CBT) and motivational interviewing. Further, these interventions can be delivered in a variety of formats (individual, family, group, electronic media) and used in different settings (eg, inpatient or community).

Legal interventions such as community treatment orders that ensure compliance with treatment by patients who are unwilling to take medication can also be considered to be part of this range of interventions. However, most of the interventions are delivered in a complex environment. Therefore, in this review, we focus mainly on two aspects of psychosocial interventions, ie, theoretical underpinning and format, highlighting the areas which have been frequently evaluated in research.

\section{Theoretical underpinning}

\section{Motivational interviewing}

This motivational intervention is aimed at increasing the probability of taking medications consistently by highlighting the advantages of adherence and developing strategies to counteract drawbacks using tools such as "decisional balance" activity in which the perceived benefits and drawbacks of taking medications are elicited. ${ }^{17}$ To address the primary drawback of side effects, an adverse event tracking form may be used to assist in recognition of side effects, along with a personalized treatment plan to counteract these. ${ }^{18}$

\section{Psychoeducational intervention}

Psychoeducation aims to educate patients and/or their families about the illness, appropriate medications, and potential side effects. It targets individuals or patient groups. The counseling sessions are delivered face to face and/or using written or audiovisual materials. This has been the mainstay of strategies to improve adherence for years; however, the results of studies do not appear to be consistently positive. Studies that have examined psychoeducation without 
adjunctive components, such as behavioral intervention or family involvement, reported that it was not efficacious in improving adherence. ${ }^{19-21}$ Nevertheless, psychoeducation provided together with family involvement seems to have better efficacy than when given to patients alone, ${ }^{21}$ and psychoeducation becomes more efficacious when other strategies are combined, such as environmental or behavioral interventions. ${ }^{19}$

\section{Compliance therapy}

Compliance therapy has elements from different theoretical underpinnings such as psychoeducation and motivational interviewing. The main component is based on CBT, because this approach helps patients to understand the connection between relapse and medication nonadherence. ${ }^{22,23}$ A number of trials have reported the efficacy of compliance therapy, mainly in schizophrenia. ${ }^{22-25}$ Most studies have shown that compliance therapy can help to improve insight, treatment acceptance, and adherence, but some studies also report less promising results with this approach. ${ }^{25,26}$

\section{Behavioral training}

Behavioral techniques are widely used to improve adherence, both as a component of other interventions and as a means of influencing positive behaviors. These techniques are designed to influence behavior through shaping, reminding (cues), or rewarding the desired behavior (reinforcement). Examples of these methods include teaching patients the skills needed to keep track of medication, using reminder methods (eg, pillboxes, calendars), simplifying the dosing schedule (ie, reducing its behavioral demands), and using rewards and reinforcement. These methods are not widely used on their own. There is some evidence that behavioral interventions are successful in promoting adherence. ${ }^{27,28}$

\section{Cognitive-behavioral therapy}

CBT is a psychotherapeutic approach that challenges patients' cognitive processes and maladaptive behaviors through goal-oriented, explicit procedures. In CBT, adherence is conceptualized as a coping behavior based on an individual's perception of their illness and their beliefs about medication. ${ }^{29}$ Using CBT to optimize treatment adherence, the aim is to help patients identify and modify negative automatic thoughts about medication. Medication-taking behavior is conceptualized as a coping behavior based on the individual's perceptions. The therapist uses guided discovery to strengthen the patient's belief that taking medication is associated with staying well and achieving goals. There is evidence from some trials that CBT also helps to improve insight, although the approach may not directly challenge the patient's beliefs about medication. ${ }^{30}$

\section{Formats and settings of interventions}

All the interventions described above can be applied in different setting and formats. The theoretical underpinnings for these interventions are applied to a varying degree in these treatments to enhance adherence. Some of these settings and formats are described below.

\section{Family interventions}

Family interventions use a variety of behavioral and educational techniques to involve family members in facilitating, administering, and supervising the taking of medication. These include psychoeducational, behavioral, and problemsolving strategies that have been employed to optimize coping on the part of families and promote better outcomes for patients. ${ }^{19}$ Of 12 family studies reviewed, only three reported significant differences in adherence. ${ }^{19}$

\section{Community care-based interventions}

Several models of community-based care have been developed to meet the diverse needs of people with severe mental illness. These interventions are generally aimed at improving engagement with mental health services. Key components of community-based interventions include the provision of a strong and supportive social network; close monitoring of mental state including the medication regimen; and provision of stable housing and other supportive services. ${ }^{19}$ Only a small proportion of studies of community care, notably those involving assertive community treatment and intensive case management models have included assessments of medication adherence. Most studies of assertive community treatment or intensive case management have involved comparisons with less intensive or standard models of care, and there is limited evidence that such interventions are associated with significantly greater medication adherence compared with controls. ${ }^{19}$ However, as mentioned earlier, these studies have not included adequate assessment of medication adherence.

\section{Legal measures}

Mental health legislation is used in many countries to detain and treat patients who pose a significant risk to themselves or others due to severe mental illness. Patients can be forced to accept treatment, which can be pharmacologic 
or psychosocial. The law in some developed countries also gives mental health practitioners the power to force compliance in the community (known as community treatment orders or assisted outpatient treatment). There is some evidence to suggest that community treatment orders reduce hospital readmissions and improve adherence with depot medication. ${ }^{31}$ These measures are difficult to evaluate specifically for improving treatment adherence, given that legal measures are mainly intended to reduce the risk of violence or self-harm.

While legal measures for inpatient admissions are more robust and have clearer boundaries, community orders are controversial for a number of legal, ethical, and empirical reasons. ${ }^{32}$ A number of studies have been reported in the literature, using either matched controls or patients serving as their own control, typically before and after community order measures. These studies have reported contradictory findings. Most show increased follow-up with outpatient services and shorter readmissions for patients on community treatment orders. ${ }^{33}$ One would expect randomized controlled trials to add some clarity to the existing evidence. However, conducting randomized controlled trials of community treatment orders is challenging for ethical and legal reasons, and only a few have been conducted. The most robust of these trials were conducted in the USA ${ }^{34}$ and more recently in the UK, ie, the Oxford Community Treatment Order Evaluation Trial. ${ }^{35}$ These trials found that community treatment orders did not reduce readmission rates over 11-12 months compared with a control. Moreover, time to readmission, total days in hospital, psychiatric symptoms, and global functioning were not significantly different. Another descriptive study from the UK reported at the same time, however, found that implementation of a community treatment order was associated with increased engagement, fewer admissions, and increased time spent outside hospital. ${ }^{36}$

\section{Pharmacologic interventions}

Most measures used to enhance adherence rely on a good therapeutic relationship, which requires that all aspects of drug treatment are carefully balanced to suit the individual patient. This includes careful drug selection with an optimal balance of efficacy and safety, switching when a treatment is not working, dose adjustment, treating side effects, simplifying the treatment regimen, and use of long-acting injections when possible. Simplifying the medication regimen can be particularly helpful for patients with cognitive impairment. These components of pharmacologic interventions are used routinely in clinical practice but are mostly not evaluated in clinical trials. One study that examined this issue found that a decrease in dosing frequency helped patients to be more adherent. $^{37}$

Long-acting injectable medication is one of the main pharmacologic methods used to improve treatment adherence. Most guidelines recommend the use of longacting injections, especially in schizophrenia, to improve treatment adherence. ${ }^{38,39}$ However, the results from randomized controlled trials and reviews on this subject give a mixed picture. For example, a recent large randomized controlled trial has shown that there was no statistically significant difference between risperidone long-acting injection and an oral formulation in preventing hospitalization. ${ }^{40} \mathrm{~A}$ meta-analysis based on 21 randomized controlled studies with the primary outcome measure of study-defined relapse at the longest time point in the study, found that long-acting injections were not significantly superior to oral antipsychotics. This was true both for primary analyses and in number of secondary analyses. Interestingly, studies using first-generation long-acting injections and those published before 1991 demonstrated the superiority of long-acting injections over oral antipsychotics, probably reflecting a cohort bias. ${ }^{41}$ These results are not supported by mirror-image and naturalistic studies. For example, Tiihonen et $\mathrm{al}^{42}$ reported that the risk of rehospitalization with long-acting injections was one third that with oral antipsychotics in a nationwide Finnish cohort.

These results may be understandable to some extent. It can be argued that patients who agree to take depot antipsychotics for long periods are more likely to comply with medication in any case, and patients who are difficult to engage are unlikely to adhere with medication, whether oral or injectable, perhaps due to the underlying nature of their illness. Patients with better adherence to treatment, less severe illness, and better cognitive capabilities might be over-represented in clinical trials. Most importantly, participation in a clinical trial could have a substantial impact on adherence per se. The real value of depot antipsychotics would be to improve treatment adherence in patients who are difficult to engage, and unfortunately studies are lacking in this population.

\section{Measurement, management, and training for medication adherence in clinical practice}

In psychiatry we are routinely faced with more complex issues when dealing with compliance. In the case of 
depression, for example, taking medication even when clinically depressed is seen by some patients as a sign of moral weakness, lack of courage, or poor resilience in the face of adversity. These issues remain poorly studied, with the behavioral aspects of taking medication being the main focus on in clinical practice. This requires that assessment and management of ongoing engagement in the treatment process becomes a central part of our clinical practice.

Maintaining engagement in treatment is a challenging task. Clients who are engaged in treatment are more likely to develop a therapeutic alliance, endorse treatment goals, and remain in treatment for longer periods. A better therapeutic alliance results in improved outcomes and better patient satisfaction. ${ }^{43}$ This essential component of effective treatment reflects the quality of the interaction, the collaborative nature of developing tasks and goals of treatment, and the personal bond between client and therapist. ${ }^{44}$ Research into determinants of the therapeutic alliance has mainly focused on psychotherapy, ${ }^{45}$ but there is also some evidence that improvement of the therapeutic alliance improves insight and medication adherence. ${ }^{46}$ Measuring the therapeutic alliance in the context of management of medication is associated with improved medication adherence. ${ }^{47} \mathrm{~A}$ recent systematic review reported that 17 of 23 studies showed positive associations with adherence. ${ }^{48}$ The authors concluded that improvement in the clinician-patient alliance and communication is associated with more favorable patient adherence. However, only four of these studies used intervention designs, and there is a need to study further the impact of the therapeutic alliance on adherence with medication.

It has been observed that clinicians generally spend too little time assessing and addressing the attitudes and behaviors which determine adherence in routine clinical practice. ${ }^{49,50}$ Assessing and maintaining treatment in the long term requires shared decision-making, which requires much more patience and time on the part of therapist. In video-recorded outpatient psychiatric visits carried out in 72 patients suffering from depression and schizophrenia, it was observed that there were two decisions made at each visit, one of which concerned medication and lasted about 2 minutes. ${ }^{49}$ Considering the central importance of shared decision-making in maintaining treatment adherence, it is surprising how little time is devoted to this activity. The real challenge is to destigmatize medication-taking in psychiatry and create a more enabling environment of education and shared decision-making while taking responsibility for managing the illness as partners in the process. $^{49}$

Adherence is not included as a topic in most textbooks of psychiatry, and most psychiatric residency programs offer little in the way of formal training on the topic of medication compliance. Formal teaching and training regarding medication adherence is considered to be an orphan topic in postgraduate training. The subject of compliance or adherence with treatment does not clearly fall within the domain of psychopharmacology, nor does it clearly fit into other formal parts of a psychiatric residency curriculum. ${ }^{50}$ When reviewing the American Board of Psychiatry and Neurology core curriculum, Weiden et $\mathrm{al}^{51}$ found no mention of medication compliance/adherence as a specific training goal. To address this omission, the authors designed and taught a five-session course for post-graduate psychiatry residents that specifically addressed assessment and management of noncompliance. This proposed curriculum covered major aspects of the theory and practice of compliance, such as definition and assessment, understanding how compliance affects efficacy, the importance of the therapeutic alliance, and pharmacologic and psychosocial strategies to improve compliance. However, this curriculum has not been widely adopted.

\section{Assessing adherence in a clinical setting}

Although a variety of measures has been used in research to measure adherence, insufficient information exists with regard to measuring adherence in the clinical setting. Most of the information regarding assessment of adherence is based on clinical experience or limited research. Clinicians can start by asking patients "Have you missed any pills in the past week?" A response of missing one or more pills indicates there may be a problem with adherence. Clinicians should bear in mind that patients tend to overestimate their actual adherence with therapy and that the accuracy of the selfreport depends on the patient's cognitive abilities, attitudes, and openness towards the therapist.

It is important to assess adherence in every follow-up appointment. Clinicians can ask several simple questions to assess the degree of adherence with the treatment. It is important to focus not only on drug-taking behavior, but also on the side effects and problems associated with treatment. Some simple questions that can be asked in routine clinical care include the following: "How are you taking your medications?", "Have you ever forgotten to take your medications?", "Are you experiencing any adverse drug reactions?", and "How are you feeling since you started 
your medication?" Patient adherence to medication can also be assessed by asking patients to report the number of days they took the prescribed drug during the previous month. This self-reported information can be adequate in clinical practice to assess adherence with medication.

\section{Engaging patients and improving adherence}

Torem $^{52}$ argues that engaging patients as partners in treatment can improve outcomes. Successful engagement with treatment is the key to good adherence. It is dependent upon a good therapeutic relationship with patients. Torem emphasizes the importance of exploring the patient's views about treatment, which can lead to a collaborative approach in the choice of medication, dosage, delivery, and the frequency of its use. They assert helping patients to take a more active role in monitoring medication and management of illness. These ideas are based in routine clinical observations and have been suggested by others, to improve adherence in patients with physical health problems. For example, Harmon et al, ${ }^{53}$ have suggested that in order to improve adherence in patients with hypertension, providers should identify barriers to medication adherence and actively engage patients in shared decision-making regarding their treatment at the start of the treatment.

It is often not realized that with a good therapeutic relationship, a patient may engage with a therapist, but not with the medication. Taking long-term medication with the burden of considerable side effects is a major undertaking and physicians need to closely monitor and encourage patients to take medications. Strategies such as simplifying the treatment regimen, frequent discussions about the link between the illness severity and treatment benefits, providing oral and written instructions, use of practical medication reminders like calendars or dose counters etc, and tailoring the treatment to fit the patient's lifestyle, can help to improve engagement with the treatment. Finally, it must be recognized that not very strategy works in all patients, and clinicians need to develop a multicomponent approach for different patients.

\section{Public health aspects of treatment adherence in psychiatry}

Psychiatry has generally overlooked the public health aspect of poor adherence with treatment, which not only includes increased complications for patients, but is also associated with a huge burden of illness resulting from violence, suicide, and substance abuse. Faced with a similar challenge, other disciplines have adopted a public health approach.
In conditions such as human immunodeficiency virus and tuberculosis, public health measures to improve free access to treatment have resulted in huge improvements in treatment outcome. ${ }^{12}$ Even for noncommunicable disorders such as diabetes mellitus, public health approaches have been adopted to improve access to treatment. In several countries, including many low-income communities, insulin is provided free of charge to enhance treatment adherence by overcoming cost-related barriers. Tuberculosis control programs worldwide have adopted the DOTS strategy, and one of the essential elements of this is to ensure a regular uninterrupted supply of all essential antituberculosis drugs backed by governmental commitment. The DOTS program has significantly reduced nonadherence with treatment in most developing countries, and is considered to be one of the most cost-effective health interventions. $^{12}$

We have argued that similar programs are required to address structural barriers to optimum treatment adherence in resource-poor settings, where the treatment gap in schizophrenia is estimated to be $70 \%-90 \%$. It is suggested that resources need to be mobilized to create a global fund for free medicines targeting the initial 2 years in the course of schizophrenia, if not the whole course of the illness. ${ }^{54}$ This would help to overcome nonadherence with treatment during this "critical period" in the disease course, which is the strongest predictor of long-term outcome and disability.

\section{Conclusion}

Most of the literature on nonadherence in psychiatry focuses on psychotic disorders, but information on how to improve adherence in nonpsychotic disorders is sparse. A number of psychosocial interventions have been proposed and studied, and a large number of randomized controlled trials have evaluated a variety of interventions to improve adherence. Some have examined a specific intervention, while others have examined the combination of two or more types of interventions. ${ }^{3}$ The outcome measures of these interventions vary widely, and they include a variety of theoretical backgrounds, formats, and settings. It is difficult to draw clear lines between interventions and to categorize them in specific groups, but the key components include psychoeducation, CBT, and motivational interviewing. These interventions are delivered in a variety of health care settings and can be used under legal powers to enhance engagement with services. The results are mixed, but interventions specifically designed to improve adherence with a more intensive and focused 
approach, interventions combining elements from different areas such as CBT-based, family-based, and community-based approaches, have shown consistently favorable results. ${ }^{19}$ The results of pharmacologic interventions are not clear. Given the discrepancy between the results obtained from randomized controlled trials, nationwide cohort studies, and mirror-image studies, the question arises as to what is the best way of assessing the effectiveness of long-term injections in comparison with oral medication.

Although it is generally accepted from a clinical point of view that adherence is of utmost importance, the topic is not covered adequately in psychiatric training and textbooks. Strategies to measure and improve adherence in clinical practice are based more on personal experience rather than evidence. Clinicians generally spend too little time discussing and addressing attitudes and behaviors regarding adherence. There is a need to include training on improving adherence in the psychiatric curriculum, both at the graduate and postgraduate levels. Future research should also focus on nonadherence in nonpsychotic disorders and the effectiveness of long-term injections in patients who are difficult to engage in treatment.

\section{Disclosure}

The authors report no conflicts of interest in this work.

\section{References}

1. Haynes R, Yao X, Degani A, Kripalani S, Garg A, McDonald H. Interventions to enhance medication adherence. Cochrane Database Syst Rev. 2005:1-96.

2. Osterberg L, Blaschke T. Adherence to medication. $N$ Engl J Med. 2005;353:487-497.

3. Lacro JP, Dunn LB, Dolder CR, Leckband SG, Jeste DV. Prevalence of and risk factors for medication nonadherence in patients with schizophrenia: a comprehensive review of recent literature. J Clin Psychiatry. 2002;63:892-909.

4. Hess LM, Raebel MA, Conner DA, Malone DC. Measurement of adherence in pharmacy administrative databases: a proposal for standard definitions and preferred measures. Ann Pharmacother. 2006;40:1280-1288.

5. Gilmer TP, Dolder CR, Lacro JP, et al. Adherence to treatment with antipsychotic medication and health care costs among Medicaid beneficiaries with schizophrenia. Am J Psychiatry. 2004;161:692-699.

6. Jónsdóttir H, Opjordsmoen S, Birkenaes AB, et al. Medication adherence in outpatients with severe mental disorders: relation between self-reports and serum level. J Clin Psychopharmacol. 2010;30:169-175.

7. Velligan DI, Wang M, Diamond P, et al. Relationships among subjective and objective measures of adherence to oral antipsychotic medications. Psychiatr Serv. 2007;58:1187-1192.

8. Valenstein M, Ganoczy D, McCarthy JF, Myra Kim H, Lee TA, Blow FC. Antipsychotic adherence over time among patients receiving treatment for schizophrenia: a retrospective review. J Clin Psychiatry. 2006;67:1542-1550.

9. Lingam R, Scott J. Treatment nonadherence in affective disorders. Acta Psychiatr Scand. 2002;105:164-172.

10. Johnson RE, McFarland BH. Lithium use and discontinuation in a health maintenance organization. Am J Psychiatry. 1996;153:993-1000.
11. Whooley MA, Simon GE. Managing depression in medical outpatients. N Engl J Med. 2000;343:1942-1950.

12. Farooq S, Nazar Z, Irfan M, et al. Schizophrenia treatment adherence in a resource-poor setting: randomised controlled trial of Supervised Treatment in Outpatients for Schizophrenia (STOPS). Br J Psychiatry. 2011;199:467-472.

13. Bull SA, Hu XH, Hunkeler EM, et al. Discontinuation of use and switching of antidepressants influence of patient-physician communication. JAMA. 2002;288:1403-1409.

14. Boudreau DM, Capoccia KL, Sullivan SD, et al. Collaborative care model to improve outcomes in major depression. Ann Pharmacother. 2002;36:585-591.

15. Kane JM, Perlis RH, DiCarlo LA, Au-Yeung K, Duong J, Petrides G. First experience with a wireless system incorporating physiologic assessments and direct confirmation of digital tablet ingestions in ambulatory patients with schizophrenia or bipolar disorder. J Clin Psychiatry. 2013;74:e533-e540.

16. Herz MI, Lamberti S, Mintz J, et al. A program for relapse prevention in schizophrenia. A controlled study. Arch Gen Psychiatry. 2000;57: 277-283.

17. Miller WR, Conforti K, Rollnik S. Motivational Interviewing: Preparing People for Change. 2nd ed. New York, NY, USA: The Guilford Press; 2002.

18. Patterson TL, Mausbach BT, McKibbin C, Goldman S, Bucardo J, Jeste DV. Functional adaptation skills training (FAST): a randomized trial of a psychosocial intervention for middle-aged and older patients with chronic psychotic disorders. Schizophr Res. 2006;86:291-299.

19. Zygmunt A, Olfson M, Boyer CA, Mechanic D. Interventions to improve medication adherence in schizophrenia. Am J Psychiatry. 2002;159:1653-1664.

20. Lincoln TM, Wilheim K, Nestoriuc Y. Effectiveness of psychoeducation for relapse, symptoms, knowledge, adherence and functioning in psychotic disorders: a meta-analysis. Schizophr Res. 2007;96: 232-245.

21. Xia J, Merinder LB, Belgamwar MR. Psychoeducation for schizophrenia. Cochrane Database Syst Rev. 2011;6:CD002831.

22. Kemp R, Hayward P, Applewhaite G, et al. Compliance therapy in psychotic patients: randomized controlled trial. BMJ. 1996;312: 345-349.

23. Kemp R, Kirov G, Everitt B, Hayward P, David A. Randomised controlled trial of compliance therapy: 18-month follow-up. $\mathrm{Br} \mathrm{J}$ Psychiatry. 1998;172:413-419.

24. Merinder LB, Viuff AG, Laugesen HD, Clemmensen K, Misfelt S, Espensen B. Patient and relative education in community psychiatry: a randomized controlled trial regarding its effectiveness. Soc Psychiatry Psychiatr Epidemiol. 1999;34:287-294.

25. Gray R, Leese M, Bindman J, et al. Adherence therapy for people with schizophrenia. European multicentre randomised controlled trial. $\mathrm{Br} J$ Psychiatry. 2006;189:508-514.

26. O’Donnell C, Donohoe G, Sharkey L, et al. Compliance therapy: a randomized controlled trial in schizophrenia. BMJ. 2003;327:834.

27. Peterson AM, Takiya L, Finley R. Meta-analysis of trials of interventions to improve medication adherence. Am J Health Syst Pharm. 2003;60:657-665.

28. Haynes RB, Ackloo E, Sahota N, McDonald HP, Yao X. Interventions for enhancing medication adherence. Cochrane Database Syst Rev. 2008;2:CD000011.

29. Scott J. Cognitive and behavioral approaches to medication adherence. Adv Psychiatr Treat. 1999;5:338-347.

30. Vita A, Barlati S, Sacchetti E. Non pharmacological strategies to enhance adherence and continuity of care in schizophrenia. In: Sacchetti E, Vita A, Siracusano A, Fleischhacker W, editors. Adherence to Antipsychotics in Schizophrenia. Milan, Italy: Springer-Verlag; 2014.

31. Vaughan K, McConaghy N, Wolf C, Myhr C, Black T. Community treatment orders: relationship to clinical care, medication compliance, behavioural disturbance and readmission. Aust $N Z J$ Psychiatry. 2000;34:801-808. 
32. Smith LS, Dawson J, Burns T. Supervised community treatment just as legitimate as compulsory hospital care. Br J Psychiatry. 2008;193:96-100.

33. Rugkåsa J, Dawson J. Community treatment orders: current evidence and the implications. Br J Psychiatry. 2013;203:406-408.

34. Swartz MS, Swanson JW, Wagner HR, Burns BJ, Hiday VA, Borum R. Can involuntary outpatient commitment reduce hospital recidivism? Findings from a randomized trial with severely mentally ill individuals. Am J Psychiatry. 1999;156:1968-1975.

35. Burns T, Rugkåsa J, Molodynksi A, et al. Community treatment orders for patients with psychosis: a randomised controlled trial (OCTET). Lancet. 2013;381:1627-1633.

36. Dye S, Dannaram S, Loynes B, Dickenson B. Supervised community treatment: 2-year follow-up study in Suffolk. Psychiatrist. 2012;36: 298-302.

37. Pfeiffer PN, Ganoczy D, Valenstein M. Dosing frequency and adherence to antipsychotic medications. Psychiatr Serv. 2008;59: 1207-1210.

38. Velligan DI, Weiden PJ, Sajatovic M, et al. The expert consensus guideline series: adherence problems in patients with serious and persistent mental illness. J Clin Psychiatry. 2009;70(Suppl 4):1-46.

39. National Institute for Health and Care Excellence. Core interventions in the treatment and management of schizophrenia in primary and secondary care (update). Leicester, UK: British Psychological Society and Royal College of Psychiatrists; 2009. Available from: http:// www.nice.org.uk/nicemedia/pdf/CG82NICEGuideline.pdf. Accessed February 14, 2014

40. Rosenheck RA, Krystal JH, Lew R; CSP555 Research Group. Long-acting risperidone and oral antipsychotics in unstable schizophrenia. N Engl J Med. 2011;364:842-851.

41. Kishimoto T, Robenzadeh A, Leucht C, et al. Long-acting injectable vs oral antipsychotics for relapse prevention in schizophrenia: a metaanalysis of randomized trials. Schizophr Bull. 2014;40:192-213.

42. Tiihonen J, Haukka J, Taylor M, et al. A nationwide cohort study of oral and depot antipsychotics after first hospitalization for schizophrenia. Am J Psychiatry. 2011;168:603-609.

43. Couture SM, Roberts DL, Penn DL, Cather C, Otto MW, Goff D. Do baseline characteristics predict the therapeutic alliance in the treatment of schizophrenia? J Nerv Ment Dis. 2006;194(1):10-14
44. Digiuseppe R, Linscott J, Jilton R. Developing the therapeutic alliance in child-adolescent psychotherapy. Appl Prev Psychol. 1996;5: 85-100.

45. Junghan UM, Leese M, Priebe S, Slade M. Staff and patient perspectives on unmet need and therapeutic alliance in community mental health services. Br J Psychiatry. 2011;191:543-547.

46. Misdrahi D, Petit M, Blanc O, Bayle F, Llorca PM, Perrens C. The influence of therapeutic alliance and insight on medication adherence in schizophrenia. Nord J Psychiatry. 2011;66:49-54.

47. Pringle J, Melczak M, Aldridge A, Snyder M, Smith R. Medication adherence and its relationship to the therapeutic alliance: results from an innovative pilot study within a community pharmacy MTM practice. Innov Pharm. 2011;2:1-14.

48. Thompson L, McCabe R. The effect of clinician-patient alliance and communication on treatment adherence in mental health care: a systematic review. BMC Psychiatry. 2012;12:87.

49. McCabe R, Khanom H, Bailey P, et al. Shared decision-making in ongoing outpatient psychiatric treatment. Patient Educ Couns. 2013;91: 326-328.

50. Kane JM, Kishimoto T, Correll CU. Non-adherence to medication in patients with psychotic disorders: epidemiology, contributing factors and management strategies. World Psychiatry. 2013;12:216-226.

51. Weiden P, Rao N. Teaching medication compliance to psychiatric residents: placing an orphan topic into a training curriculum. Acad Psychiatry. 2005;29:203-210.

52. Torem SM. Participatory pharmacotherapy: 10 strategies for enhancing adherence. Curr Psychiatry. 2013;12:21-25. Available from http://www.currentpsychiatry.com/topics/practice-trends/article/ participatory-pharmacotherapy-10-strategies-for-enhancing-adherence. html. Accessed March 11, 2014.

53. Harmon G, Lefante J, Krousel-Wood M. Overcoming barriers: the role of providers in improving patient adherence to antihypertensive medications. Curr Opin Cardiol. 2006;21(4):310-315.

54. Farooq S. Public health and physician focused strategies to improve medication adherence in psychotic disorders. World Psychiatry. $2013 ; 12: 258-240$
Neuropsychiatric Disease and Treatment

\section{Publish your work in this journal}

Neuropsychiatric Disease and Treatment is an international, peerreviewed journal of clinical therapeutics and pharmacology focusing on concise rapid reporting of clinical or pre-clinical studies on a range of neuropsychiatric and neurological disorders. This journa is indexed on PubMed Central, the 'PsycINFO' database and CAS

\section{Dovepress}

The manuscript management system is completely online and includes a very quick and fair peer-review system, which is all easy to use. Visit http://www.dovepress.com/testimonials.php to read real quotes from published authors. 